\title{
A First Sign Not to be Missed: Cutaneous Metastasis from Breast Cancer
}

\author{
Elsa Araújo, Manuel Barbosa, Raquel Costa, Bárbara Sousa, Vítor Costa \\ Internal Medicine Department, Unidade Local de Saúde do Alto Minho, Ponte de Lima, Portugal
}

\section{Doi: 10.12890/2020_001356 - European Journal of Case Reports in Internal Medicine - @ EFIM 2020}

Received: 30/10/2019

Accepted: 04/11/2019

Published: 08/01/2020

How to cite this article: Araújo E, Barbosa M, Costa R, Sousa B, Costa V. A first sign not to be missed: cutaneous metastasis from breast cancer. EJCRIM 2020;7: doi:10.12890/2020_001356.

Conflicts of Interests: The Authors declare that there are no competing interest

This article is licensed under a Commons Attribution Non-Commercial 4.0 License

\section{ABSTRACT}

Cutaneous metastasis has a frequency of 1 to $10 \%$ among all metastatic cancer forms and breast cancer accounts for $30 \%$ of all cases. We report the case of a 73-year-old woman who presented with 4 skin lesions distributed across the upper trunk and abdomen; these had developed over a period of 12 months. Over the previous 6 months she had also developed anorexia, asthenia and weight loss. Upon investigation, a nodular mass was found in the left breast. Skin and breast mass biopsy were performed. Histology confirmed the diagnosis: infiltrating lobular breast cancer with cutaneous metastasis. The patient underwent hormonal treatment, mastectomy and radiotherapy. In rare cases, cutaneous metastasis appears as the first clinical manifestation of breast cancer. It is therefore crucial for patients and health professionals alike to be aware of new skin lesions. Cutaneous metastasis is a diagnostic sign of cancer that, it must be emphasised, is not restricted exclusively to later forms of the disease.

\section{LEARNING POINTS}

- Although breast cancer has the highest cutaneous metastasis rate among internal malignancies, cutaneous metastases actually occur in only a small minority of breast cancer patients. In rare cases, skin metastasis may appear as the first clinical manifestation of breast cancer.

- The clinical presentation of cutaneous metastasis from breast cancer can be varied, and therefore warrants greater awareness in clinical practice.

- Cutaneous metastasis from breast cancer typically carries a less unfavourable prognosis than cutaneous metastasis from other internal malignancies.

\section{KEYWORDS}

Skin lesions, cutaneous metastases, metastatic breast carcinoma, breast cancer

\section{CASE DESCRIPTION}

We report the case of a 73-year-old woman with a history of hypertension and type 2 diabetes mellitus. She had no past or current history of smoking or alcoholism, and no relevant family history.

Presenting with asthenia, anorexia and weight loss, which had developed over 6 months, she was referred to an outpatient internal medicine facility.

The patient expressed concern about 4 skin lesions, present for more than a year. These were small, violet and scattered across the upper trunk and abdomen, with no other accompanying symptoms (Fig. 1). Her attending physician had suspected a skin infection or allergic reaction and prescribed topical antifungal and corticosteroid medication without any remarkable effect. 
In addition to the six-month history of asthenia, anorexia and weight loss ( $20 \%$ of her body weight), she also reported bouts of diarrhoea and abdominal pain.

On examination, she was found to have a body mass index of $23.4 \mathrm{~kg} / \mathrm{m}^{2}$; she had bleached mucous membranes and four erythematous, violet annular plaque lesions measuring between 1 and $4 \mathrm{~cm}$ - one in the right anterior axillary region (Fig. 2), two in the right inframammary region and one in the umbilical region. Breast examination revealed a $2 \mathrm{~cm}$ nodular mass in the external lower quadrant of the left breast. No adenomegalies were observable. A dermatology specialist was asked to observe the lesions, having first performed a skin biopsy. The differential diagnosis included cutaneous lymphoma, Jessner's lymphocytic infiltrate, specific leukocyte infiltrate and cutaneous metastasis.
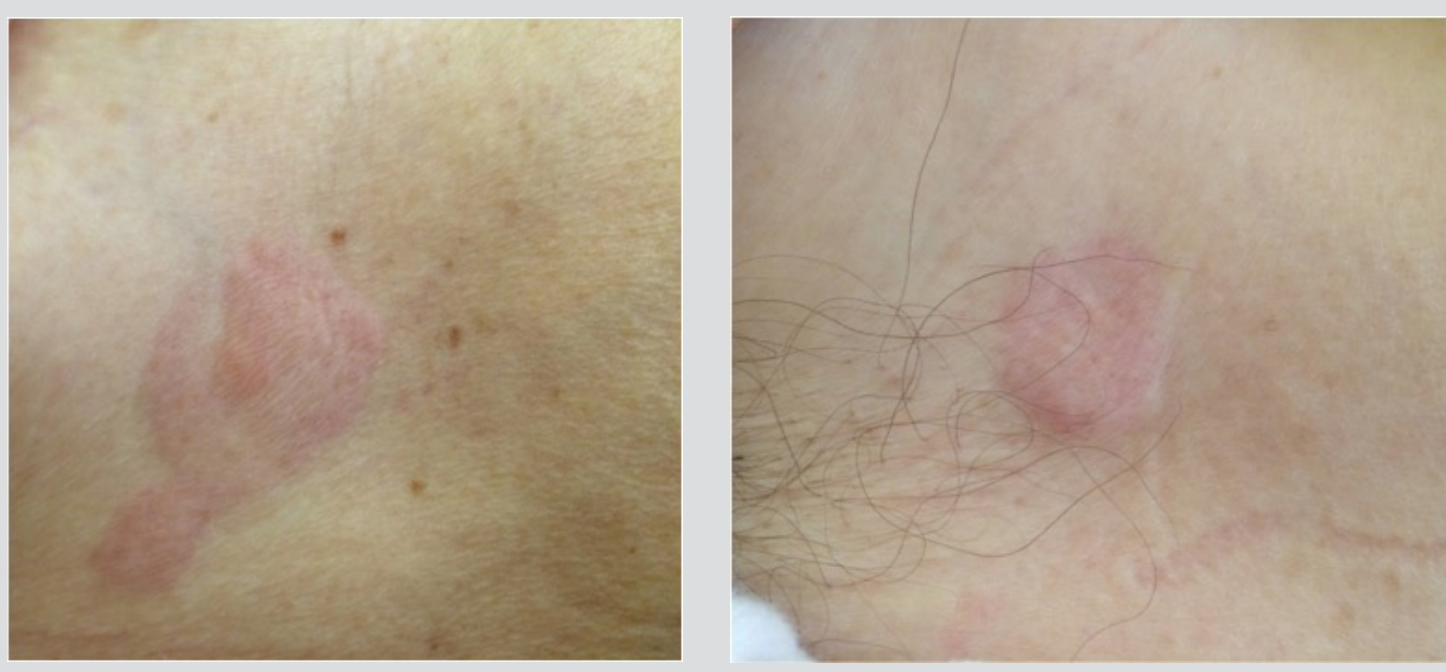

Figure 1. Violet erythematous annular plaque in the right inframammary region

Figure 2. Violet erythematous annular plaque in the right anterior axillary region

The patient underwent breast ultrasound and mammography, which confirmed the presence of a heterogeneous nodular lesion, with a maximum diameter of about $2 \mathrm{~cm}$, in the lower external quadrant of the left breast, and left axillary adenopathy. A biopsy was performed. The remaining diagnostic examinations revealed anaemia with a haemoglobin level of $9.2 \mathrm{~g} / \mathrm{dl}$ and an increased erythrocyte sedimentation rate $(86 \mathrm{~mm} / \mathrm{h})$. Thoracic, abdominal and pelvic computed tomography (CT) imaging revealed mammary asymmetry due to higher density of the left breast and parietal thickening at the level of the gastric antrum and pylorus. Given the gastrointestinal complaints and CT scan findings, it was decided to perform an oesophagogastroduodenoscopy to evaluate possible gastric injury. This examination revealed the presence of erythema in the notch and gastric antrum. Biopsies were performed and Helicobacter pylori infection was identified, for which the patient was treated.

Histopathological examination of the skin biopsy showed dermal infiltration with all cells expressing CAM 5.2, containing oestrogen and progesterone receptors, and cells negative for c-erbB-2 ; these findings confirmed the diagnosis of CM from breast cancer The breast mass biopsy result confirmed the diagnosis of infiltrating lobular breast cancer with the following features: positive oestrogen and progesterone receptors, c-erbB-2 negative and Ki-67 positive in 25\% of the cells, cadherin E negative and CK19 positive. Cytology of the left axillary adenopathy showed atypical cellularity (Fig. 3 and 4). Bone scintigraphy showed no bone lesions.

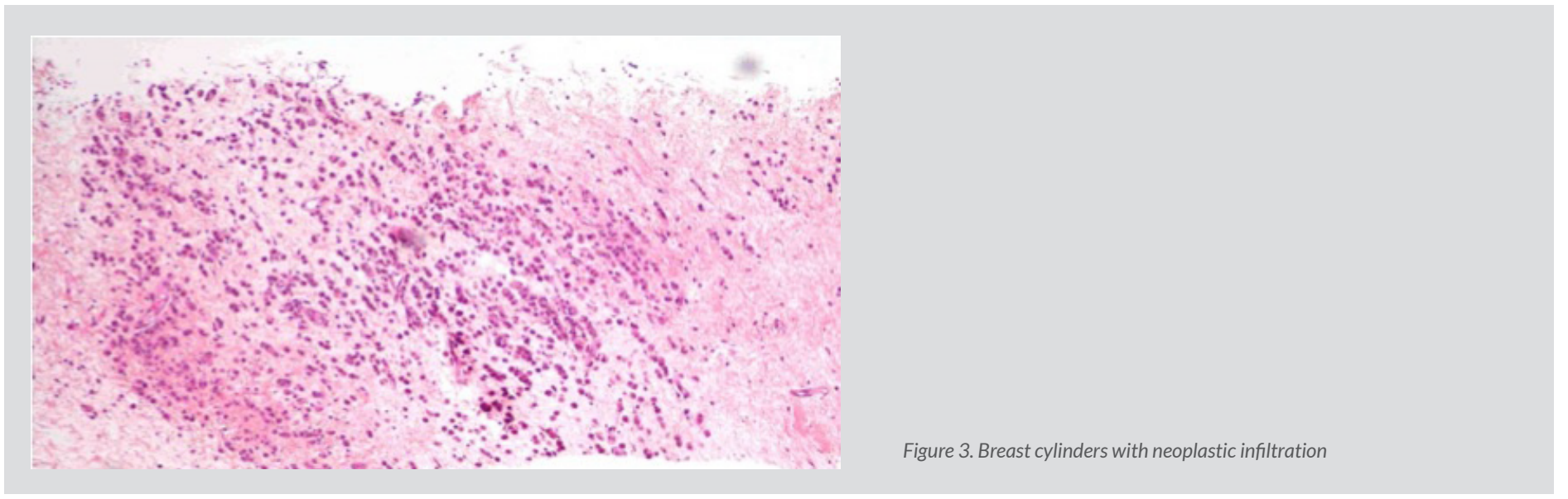


The patient was referred to oncology with the diagnosis of infiltrating lobular breast cancer with CM, Bloom's grade II (T3; P2; M1) without other distant metastasis sites. The patient started letrozole hormone therapy, and underwent bilateral mastectomy with lymph node removal and radiotherapy.

\section{DISCUSSION}

Cutaneous metastasis is an unusual clinical finding. A review of the literature indicates that the incidence of $\mathrm{CM}$ ranges from $0.7 \%$ to $10 \%$ and that $\mathrm{CM}$ accounts for $2 \%$ of cutaneous tumours. CM is more common in older individuals, paralleling the increasing incidence of malignancy with increasing age ${ }^{[1]}$. CM from solid tumours in general occurs at a rate of about $1 \%$ per primary tumour. In breast cancer, $\mathrm{CM}$ occurs at a rate of about $2.5 \%$ per primary tumour. Because of its high incidence relative to other internal malignancies, breast cancer accounts for almost $33 \%$ of all cases of CM. That said, CM occurs in only a small minority of breast cancer patients ${ }^{[2]}$.

Cutaneous malignancy is defined as a neoplastic lesion affecting the dermis or the subcutaneous tissue that originates from another primary tumour ${ }^{[3]}$. Cancers can spread through vascular or lymphatic routes, by contiguous growth or by iatrogenic implantation ${ }^{[1]}$.

Cutaneous malignancies usually occur in a clinical setting of known widespread metastatic disease, and may occasionally be the first clinical manifestation of an internal malignancy, or the first evidence of recurrence of a previously treated tumour; they have a significant prognostic value, substantially reducing survival rates ${ }^{[1,3]}$.

Metastatic tumours involving the skin include carcinomas, melanomas, sarcomas and cutaneous involvement in haematopoietic malignancies ${ }^{[1]}$. About $60 \%$ of metastatic cancers are adenocarcinomas ${ }^{[3]}$. The most common primary sites in metastatic adenocarcinomas involving the skin are the breast, lung and large intestine. Other less common sites include the stomach, prostate, pancreas, ovary, endometrium and thyroid.

The type of malignancy metastasising to the skin in patients of different age and gender generally parallels the incidence of various tumours in each particular age and gender group. In adult women, CMs most commonly originate from breast cancer, melanoma, colorectal cancer and lung cancer; in men, the most common primary forms are lung cancer, melanoma, colorectal cancer and prostate cancer. In children, they are neuroblastoma and rhabdomyosarcoma ${ }^{[1]}$.

With most tumour types, CMs develop months to years after the primary tumour was initially diagnosed and in approximately $7 \%$ of cases this interval is greater than 5 years ${ }^{[1,3]}$. CMs originating from cancer of the lung, stomach, ovary and kidney have relatively frequently been diagnosed before the primary tumour, while this occurs more rarely in squamous cell carcinoma of the oral cavity and in only $3 \%$ of cases in breast cancer ${ }^{[4]}$ Our patient belongs to this rare group of patients in whom CMs were recognised before the primary tumour.

Determining the site of the primary tumour, if unknown, is often very difficult and sometimes impossible. However, certain primary sites may be suspected from the location of the metastases ${ }^{[1]}$. It has been reported that in most patients the lesions were distributed in a single anatomical region, while multiple areas were involved in only $3 \%$ of patients at the time of diagnosis ${ }^{[4]}$ The upper trunk and the abdomen were the most frequent sites of metastases, followed by the head and neck. The umbilicus was also a common site. Metastases to the extremities were uncommon. Lung cancer tends to metastise to the head, neck and trunk, and colon cancer to the abdomen ${ }^{[5]}$. Breast cancer tends to metastasise to the chest, abdomen and scalp; less frequently to the back, upper arms and lower abdomen; and rarely to the buttocks, perianal region, lower extremities and eyelids ${ }^{[6]}$. 
The clinical presentation of CMs is reported to be highly variable ${ }^{[5]}$. Most commonly they manifest as multiple, discrete, painless, mobile nodules of sudden onset. Most lesions are less than $2 \mathrm{~cm}$ in diameter at presentation. Solitary metastases occur in approximately $10 \%$ of cases and are usually indistinguishable clinically from other skin lesions, particularly primary tumours. A CM may occasionally present as a plaque, an area of alopecia or resemble a dermatological condition such as erythema annulare, lupus erythematosus, herpes zoster or a condyloma ${ }^{[1]}$. It is thus clear that CMs can often mimic other clinical entities ${ }^{[5]}$.

The following signs and symptoms may or may not be present in any combination: warmth, pruritus, erythema, pain, stinging, macular rash and thickening or hardening of the skin ${ }^{[6]}$.

Metastatic skin lesions deriving from breast cancer are usually asymptomatic measure $1-3 \mathrm{~cm}$, and appear as firm, pink to red-brown nodules on the chest ipsilateral to the primary tumour. However, their appearance can vary and unusual cutaneous presentations include multiple telangiectatic papules, peau d'orange and carcinoma en cuirasse ${ }^{[1]}$. Our patient did not have the most common clinical features. First, here CMs were distributed over two body areas, namely the umbilical region, an uncommon site of CMs deriving from breast cancer, and second she presented lesions located contralaterally to the site of the primary tumour.

A skin biopsy is mandatory to diagnose CM. Histological features vary depending on the type of primary malignancy and, in some instances, require differentiation from a primary cutaneous tumour. In the absence of a pertinent clinical history, it can be difficult to ascertain the primary site of metastatic cancers ${ }^{[5]}$. However, metastases usually show histopathological similarities to the primary tumour ${ }^{[1]}$. Despite through investigation with imaging techniques and immunohistochemistry, the primary tumour location cannot be determined in 5-10\% of cases.

Breast cancer immunohistochemistry reveals the $\mathrm{CK} 7+/ \mathrm{CK} 20$ - cytokeratin pattern; furthermore, oestrogens and progesterone receptors are markers that increase breast cancer detection sensitivity ${ }^{[3]}$.

Cutaneous metastatic lesions are easily and often misdiagnosed as benign lesions. It is important to recognise them promptly in order to avoid prolonged empirical anti-inflammatory therapy that will delay the correct diagnosis. Therefore, atypical or persistent nodular lesions in patients with a history of systemic malignancy or suspected malignancy should be considered for biopsy to rule out metastasis ${ }^{[5]}$. As for our patient, for more than one year she presented skin lesions that did not arouse any suspicion in her attending physician.

The period between the diagnosis of $\mathrm{CM}$ and death has been found to range from 0.25 to 50 months (median, 5 months) ${ }^{[5]}$. Metastases confined to the skin are often indolent, and patients can be maintained with tolerable treatment regimens for a number of years ${ }^{[6]}$. Breast cancer metastases to soft tissue typically carry a better prognosis than breast cancer metastases to visceral organs or bone. Furthermore, $\mathrm{CMs}$ from breast cancer do not necessarily have as poor a prognosis as CMs from other internal malignancies. CMs from other internal malignancies carry a 4.3-fold increased relative risk of mortality compared with CMs from breast cancer ${ }^{[2]}$.

A high index of clinical suspicion is essential for diagnosing CM lesions, because the clinical presentation can be subtle and confusing. Although CMs from malignant neoplasms of internal organs are relatively uncommon in clinical practice, it is important to consider this possibility in newly found lesions, however benign they seem. Early recognition, especially in breast cancer cases, can lead to rapid and accurate diagnosis and timely treatment.

\section{REFERENCES}

1. Scolyer RA, Murali R, Thompson JF. Cutaneous Metastases. Dermatopathology. Elsevier. 2010. 637-650.

2. Mayer JE, Maurer MA, Nguyen HT. Diffuse cutaneous breast cancer metastases resembling subcutaneous nodules with no surface changes. Cutis 2018;101:219-223.

3. Weimann ET, Botero EB, Mendes C, Santos MA, Stelini RF, Zelenika CR. Cutaneous metastasis as the first manifestation of occult malignant breast neoplasia. An Bras Dermatol 2016;91(5 suppl 1):105-107.

4. Brownstein MH, Helwig EB. Patterns of cutaneous metastasis. Arch Dermatol 1972;105:862-868.

5. Sariya D, Ruth K, Adams-McDonnell R, Cusack C, Xu X, Elenitsas R, et al. Clinicopathologic correlation of cutaneous metastases: experience from a cancer center. Arch Dermatol 2007;143:613-620.

6. Moore S. Cutaneous metastatic breast cancer. Clin J Oncol Nurs 2002;6:255-260. 